\title{
Local microwave heating of sand molds as a means to overcome design limitations in sand mold casting
}

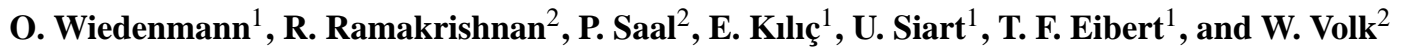 \\ ${ }^{1}$ Technische Universität München, Lehrstuhl für Hochfrequenztechnik, Arcisstr. 21, 80333 Munich, Germany \\ ${ }^{2}$ Technische Universität München, Lehrstuhl für Umformtechnik und Gießereiwesen, Walther-Meißner-Straße 4, \\ 85747 Garching, Germany \\ Correspondence to: O. Wiedenmann (oliver.wiedenmann@tum.de)
}

Received: 20 December 2013 - Revised: 25 February 2014 - Accepted: 27 March 2014 - Published: 10 November 2014

\begin{abstract}
Microwave induced selective heating outmatches conventional mold heating by convective heat transfer in means of energy efficiency and cycle time. Moreover, it provides the opportunity of a local manipulation of solidification and cooling processes within the sand casting mold. In this paper, we investigate the suitability of different highly absorbing materials to indirectly heat up the mostly microwave transparent sand mold. The temperature-dependent permittivity of the involved materials is determined by resonator experiments and subsequently used to simulate the electromagnetic field and the thermodynamic response of the sand mold prior to a metal casting process. Experimental results are presented and compared with the outcome of the coupled electromagnetic-thermodynamic simulations and the influence of local microwave heating on the solidification and cooling of the cast is studied.
\end{abstract}

\section{Introduction}

Applying microwaves during the production and processing of industrial goods is a widely accepted and popular technology. Especially in the food and ceramic industries drying and heating by microwaves outmatches conventional heating methods in many areas (Meredith, 1998). With regards to the metal casting industry microwave technology is merely used for drying and firing of ceramic mold materials (Robak, 2005). Furthermore, Chandrasekaran et al. (2011) investigated the heating of different metals by microwave energy. This paper focuses on the generation of a custom temperature field inside a sand mold by selective microwave heating.
In die casting processes temperature control by means of heating and cooling channels is commonly used and necessary to assure a controlled solidification front and thus the requested mechanical properties of the cast part. In sand mold casting, however, the options for a temperature control are limited. Only cooling elements, so called chills, or exothermic feeders are used frequently. Whereas convective heating of the mold is not reasonable, as the heat input can not be locally defined, microwave heating offers a possibility to generate an inhomogeneous temperature distribution. In this work, a custom temperature field in sand molds is achieved by positioning highly absorbing materials, so called susceptors, within the mold. Hence, we combine microwave transparent bulk materials with susceptor materials in a single sand mold. This rigged sand mold is subjected to a microwave field thus generating a locally heated sand mold prior to the casting process.

The first step is the selection of a suitable susceptor material, which fulfills all requirements for an application in a sand casting process. All involved materials are dielectrically characterized by a resonator technique in a way that allows to determine the permittivity of the material samples depending on the temperature. This knowledge is used to develop a simulation model to predict the electromagnetic and thermodynamic behavior of the experimental setup. Due to the strong temperature dependency of the permittivity of the susceptor material, electrical and thermal phenomena are coupled to each other, so that electromagnetic and thermodynamic simulations are no longer independent. Instead, a simulation model has to be derived that takes into account the coupling between the electromagnetic and the thermodynamic simulations. Finally, the results of the numerical methods and the 
casting experiments are compared and the effect of the local heating on the solidification and cooling process is investigated.

\section{Selection of susceptor materials}

The microwave experiments for the selection of susceptor materials are carried out in a laboratory microwave furnace MKE - 0.8/2.45 - 0.6/5.8 manufactured by Linn High Therm $\mathrm{GmbH}$. Microwaves are applied to the cylindrical chamber with a frequency of $2.45 \mathrm{GHz}$ which has proven to be suitable for most needs (Clark, 2005). The inner chamber diameter is $330 \mathrm{~mm}$ with a depth of $260 \mathrm{~mm}$. The packing space for the microwave furnace is specified by the manufacturer with a volume of $100 \times 100 \times 100 \mathrm{~mm}^{3}$ in the center of the cylinder.

The key to accomplish selective sand mold heating by microwave energy is the right choice of materials. The microwave energy is used to heat the susceptors, which consist of a material that shows a high microwave absorption rate. For the investigations of appropriate susceptor materials a simple two-part sand block is used, hereinafter called susceptor carrier. The bulk of the susceptor carrier, where no heating is desired, should be as microwave transparent as possible in order to convey the microwave field to the susceptors which lie within the sand. Commonly used molding sands on quartz basis meet this requirement, since these materials do not heat up significantly in a microwave field. For our experiments we manufactured the susceptor carriers from laser croning sand Resital ${ }^{\circledR}$ QLS 50 CG (HüttenesAlbertus Chemische Werke $\mathrm{GmbH}$ ) by selective laser sintering (SLS) in an EOSINT S700 (EOS GmbH) laser sintering machine. The susceptor carrier consists of a bottom block with a pocket for the susceptor material and a top block for covering the susceptor. Each bottom and top block has a size of $80 \mathrm{~mm} \times 80 \mathrm{~mm} \times 50 \mathrm{~mm}$. In the bottom part of a susceptor carrier one susceptor specimen can be positioned. Figure 1 schematically shows an example of the susceptor carrier with a susceptor specimen.

Inside the cylindrical microwave chamber a supporting surface out of aluminum oxide is positioned, which can be assumed as microwave transparent. On top of the plate the susceptor carrier including the susceptor is positioned. The presence of a metallic thermocouple inside a microwave field has a great influence on the field distribution inside the microwave chamber. Arcing and microwave interference with temperature measurement are common problems (Grellinger and Janney, 1993; Chandrasekaran et al., 2011). Therefore, thermocouples have to be inserted shortly after the microwave is turned off. The prepared susceptor carrier with one susceptor is positioned in the designated packing space of the microwave furnace. After a microwave irradiation the temperature is measured in a distance of $4 \mathrm{~mm}$ from the susceptor surface. This is the designated distance - and mini-

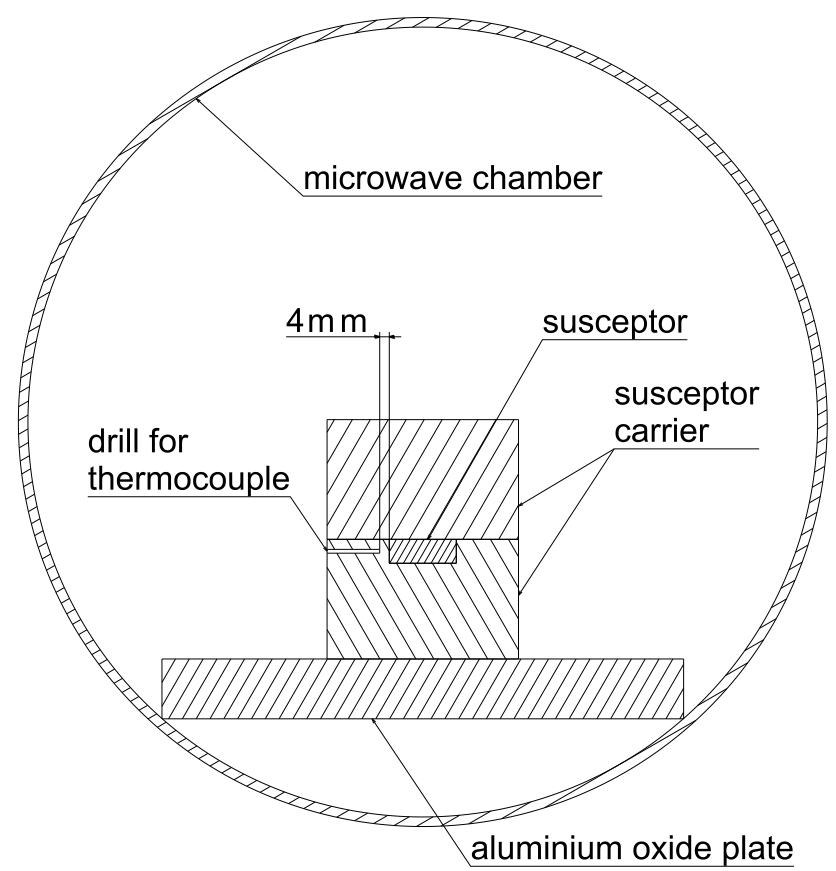

Figure 1. Scheme of the microwave chamber with susceptor carrier and susceptor positioned inside. For temperature measurements a thermocouple can be inserted when the magnetron is turned off.

mum mold wall thickness - between the susceptor and the melt in the later casting process.

As the desired microwave heat treatment of a sand mold takes place before casting, the mold stability required for a satisfying casting result has to be kept up during the irradiation. Binders in molding sands lose their stability by mechanical or thermal stress. With rising susceptor temperatures the desired effect on the casting process will be more significant. However, high susceptor temperatures increase the probability of erosion within the mold due to binder degradation. Exothermic reactions excited in susceptor materials by the microwave process can also reduce the stability of the susceptor itself or surrounding molding sand. Therefore, high reactivity of the susceptor materials has to be avoided generally, regardless of whether the susceptors are in direct contact to the casting cavity, thus shape the cast part, or surrounded by molding material.

Further demands on susceptor materials are an easy fabrication of variably shaped susceptors and that they do not represent a danger for health during fabrication, irradiation or casting. Based on a literature review the following materials are investigated as possible susceptor materials. (Rhee, 2002; Lukasz, 2005; Lühken, 2005; Bott, 1991; Gerdes et al., 1998):

- Silicon carbide $(\mathrm{SiC})$

- Tungsten carbide (WC)

- Copper oxide $(\mathrm{CuO})$ 


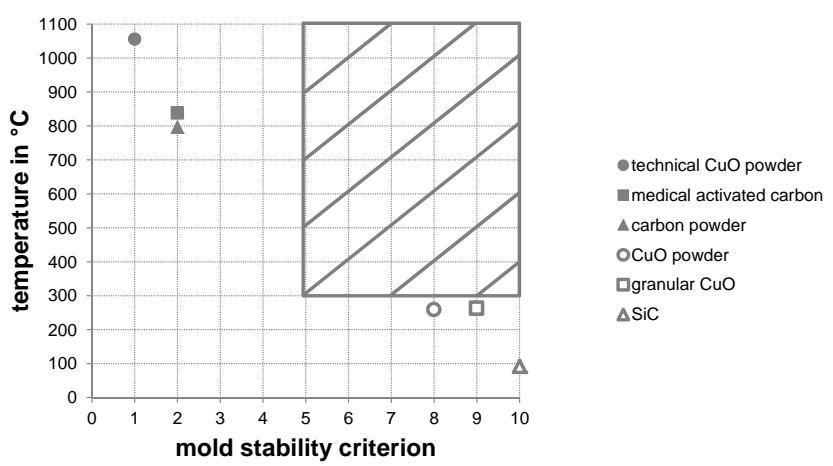

Figure 2. Maximum temperatures in $4 \mathrm{~mm}$ distance to the susceptor (pure materials) after an irradiation time of $300 \mathrm{~s}$ plotted over the mold stability.

- Graphite modifications (C)

These materials are investigated in different modifications according to the reached temperature during a defined microwave irradiation and their reactivity, which goes along with mold stability.

First the mentioned susceptor materials are used in powder form with different grain sizes. The powders are filled in the susceptor carrier pocket and irradiated for $300 \mathrm{~s}$. For the first experiments a reduced susceptor volume is used. The pocket volume filled with susceptor powder is $1000 \mathrm{~mm}^{3}$ for graphite and $2000 \mathrm{~mm}^{3}$ for other materials, preventing expected reactions of graphite.

The results of the experiments are summed up in Fig. 2. It shows the maximum temperatures measured in a distance of $4 \mathrm{~mm}$ to the susceptor materials after an irradiation time of 300 s over a mold stability criterion. This criterion was defined by visually evaluating (pairwise comparison) the damage inflicted on the sand mold in the susceptor's vicinity. Low mold stability criterion values indicate severe binder degradation and therefore strong mold disruption. To affect the solidification and cooling behavior of the cast metal significantly, an increase in temperature of $300{ }^{\circ} \mathrm{C}$ is defined as the desired lower limit. The target area, defined by the desired minimal temperature rise of $300^{\circ} \mathrm{C}$ and the acceptable mold stability criterion of 5 or higher, is highlighted.

The powder materials used in the stage 1 experiments (Fig. 2) did either not achieve the desired temperature rise of $300{ }^{\circ} \mathrm{C}$ or heated up so much, that the stability of susceptor and surrounding molding sand was destroyed and thus a defined temperature measurement in a distance of $4 \mathrm{~mm}$ was not possible. The temperatures reached in these susceptors amount to approximately $1000^{\circ} \mathrm{C}$. One reason for these high temperatures is an overlay of microwave heating and exothermal reaction. In the case of graphite, microwave irradiation induces combustion of graphite powder with surrounding air, which destroys the mold stability. In order to combine the good heating conditions reached with some materials and the required inert characteristics of others, the ini-

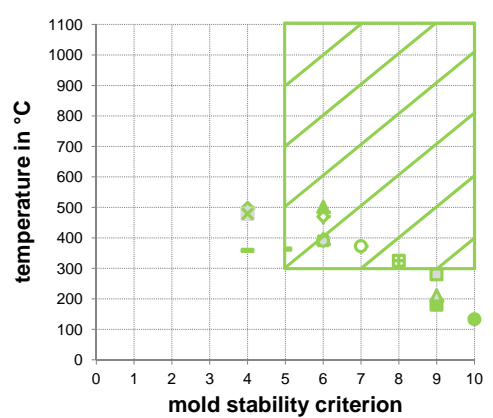

- $49 \%$ grained $\mathrm{SiC}+49 \% \mathrm{H} 32$ sand $73 \% \mathrm{H} 32$ sand $+25 \%$ WC powder $\triangle 98 \%$ grained $\mathrm{SiC}$ $49 \%$ grained $\mathrm{SiC}+49 \%$ WC powder $083 \%$ grained SiC $+15 \%$ WC powder $88 \%$ grained SiC $+6 \%$ graphite spray $\Delta 79 \%$ grained SiC $+13 \%$ graphite powder $094 \%$ grained $\mathrm{SiC}+3 \%$ graphite powder $\times 94 \%$ grained $\mathrm{SiC}+3 \%$ activated carbon $=80 \%$ grained $\mathrm{SiC}+15 \%$ green $\mathrm{SiC}$ powder $-57 \%$ grained $\mathrm{SiC}+29 \% \mathrm{SiC}$ powder $-63 \%$ grained $\mathrm{SiC}+32 \%$ techn $\mathrm{CuO}$ powder $47 \%$ grained $\mathrm{SiC}+47 \%$ wC powder $63 \%$ Sic powder $+32 \%$ carbon (36\% grand $\mathrm{SiC}+30 \%$ carbon powder $\square 60 \%$ grained SiC $+30 \%$ carbon powder $\triangle 93 \%$ WC powder mold stability criterion

Figure 3. Maximum temperatures in $4 \mathrm{~mm}$ distance to the susceptor (compressed and bound mixtures, weight balance equals binder amount) after an irradiation time of $300 \mathrm{~s}$ plotted over the mold stability.

tial materials extended by tungsten carbide are mixed in different portions and bound with varied amounts of INOTEC $®$ binder and compressed using a hand press. Silicon carbide and quartz sand (H32) were used as carrier materials, as they show low reactivity and good wettability characteristics for the INOTEC ${ }^{\circledR}$ binder. Using these mixtures, the susceptor volume could be increased to $8000 \mathrm{~mm}^{3}$ to induce a higher amount of energy without losing mold stability. Figure 3 shows the results of the corresponding experiments summarized. Considerable improvements could be reached in terms of mold stability. However silicon carbide ( $\mathrm{SiC}$ ) also showed an unexpected high maximum temperature which we attribute to the compression of the susceptor. Having one of the lowest temperatures without compression, the SiC susceptor reached the highest temperature in the stage 2 experiments. Based on this result, $\mathrm{SiC}$ bound with INOTEC ${ }^{\circledR}$ and compressed was selected as the most suitable material for susceptor applications.

\section{Material characterization by resonator experiments}

For the electromagnetic simulation, the dielectric properties of all involved materials have to be determined. Therefore, a resonator technique is employed which is based on a cylindrical cavity resonator as illustrated in Fig. 4. A sample of the material to be analyzed can be inserted into the center of the resonator. By measuring the resonance frequency and the quality factor of the detuned resonator, real and imaginary part of the permittivity of the sample material can be computed by an inversion algorithm. This approach also takes into account the effects of lossy walls and of the filling hole by imposing inhomogeneous surface impedance boundary conditions (Kilıç et al., 2013).

The method starts by converting the vector wave equation for the magnetic field into a variational form. The finite conductivity of the resonator walls is accounted for by enforcing an impedance boundary condition along the metallic surface. The filling hole, through which dielectric material 


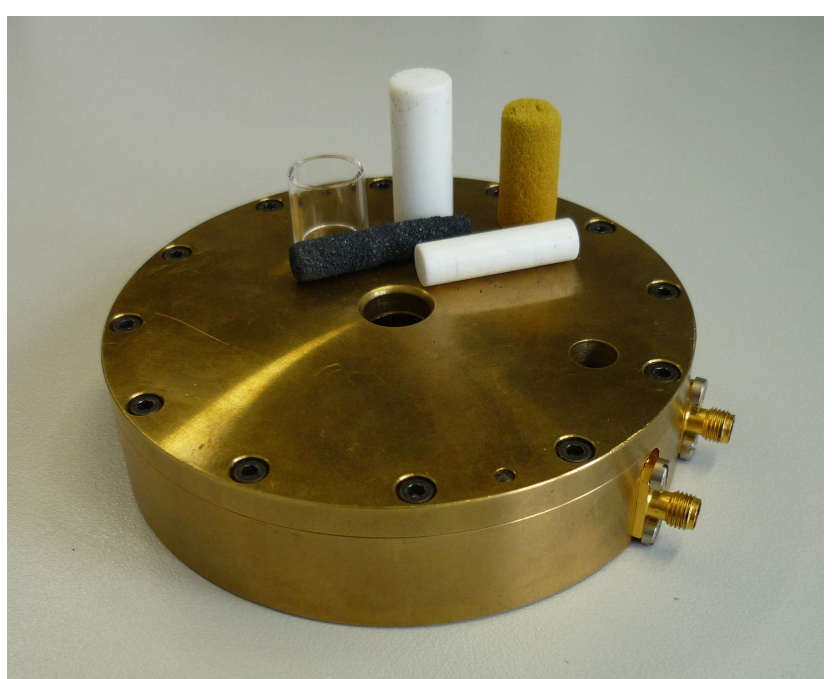

Figure 4. Cylindrical cavity resonator with an opening for the insertion of material samples.

samples can be plunged into the cavity, is modeled as a circular hollow waveguide. Therefore, the surface impedance in the cross section of the filling hole can be approximated by the scalar wave impedance of evanescent waveguide modes.

The variational expression for the magnetic field can be expanded by the natural modes of the lossless empty cavity, which form a complete set. This reduces the problem to a generalized eigenvalue problem for the unknown material parameters in matrix form. In contrast to the direct problem, in which the wave number is determined from the permittivity, the problem at hand is the other way around. Hence, it is an inverse problem, where the known quantity is the complex wave number which is determined by the measured resonance frequency and the quality factor. The inverse problem is solved by a generalized Schur decomposition. This procedure provides an accurate way to obtain the permittivity of a material sample.

The described configuration allows heating up the material sample prior to measurement, so that the temperature dependency of the permittivity can be accounted for. Thus, the temperature of the heated sample was recorded with an infrared camera during the cooling process, while the scattering parameters were measured in parallel with a vector network analyzer. The setup for the measurement of temperature and electrical scattering parameters is illustrated in Fig. 5.

Since the usage of an infrared camera only returns the temperature on the material surface, the samples have been heated up a second time, but this time the core temperature has been measured as well by inserting a thermocouple into a tiny drilled hole. This offers the possibility to assign the surface temperature measured during the resonator experiment to the corresponding core temperature. Measuring the temperature with the thermocouple during the network analyzer measurements is not feasible, because the metallic ther-

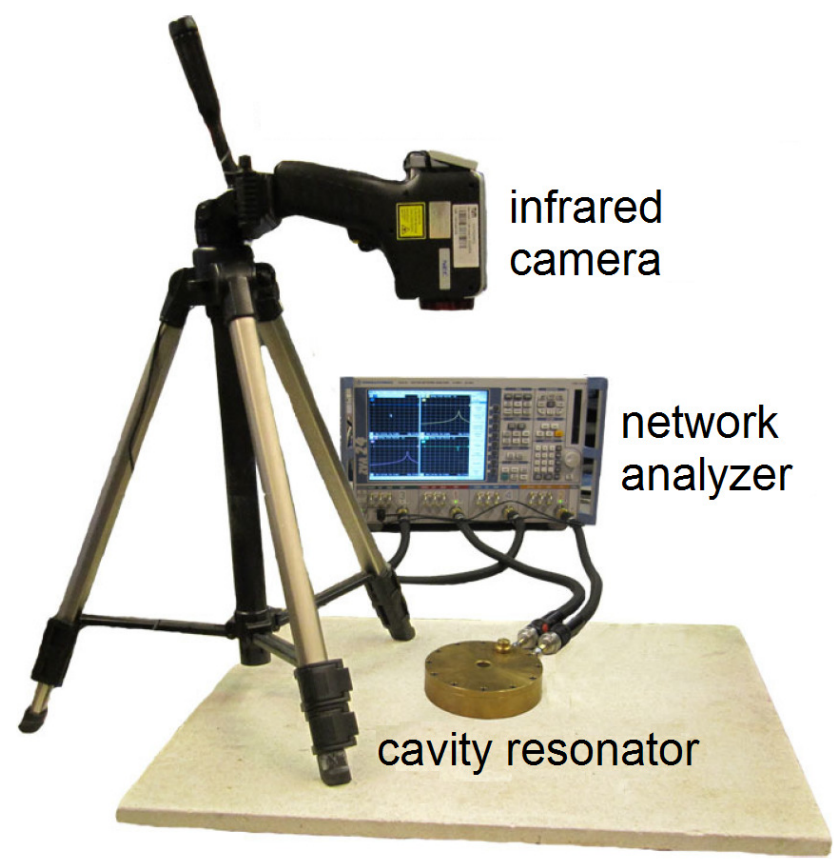

Figure 5. Measurement setup for the simultaneous determination of temperature and electrical scattering parameters comprising a network analyzer and an infrared camera.

mocouple disturbs the fields and, thus, renders the measured electric data useless.

The results for the complex relative permittivity $\varepsilon_{\mathrm{r}}^{\prime}-j \varepsilon_{\mathrm{r}}^{\prime \prime}$ of silicon carbide are illustrated in Fig. 6. It can be discerned that there is a distinctive dependency on the temperature, which has to be taken into account in the numerical simulation. A rational function is used to interpolate between the measurement points, thereby creating a functional dependency between temperature and permittivity, which is required as input to simulate the electric field distribution.

\section{Numerical simulation of electric field and heat distribution}

In order to compute the expected temperature distribution inside the sand mold after microwave heating, an electromagnetic full-wave simulation is necessary. The calculated electric field serves as input for a subsequent thermodynamic simulation which computes the heat flow between the susceptors and the sand mold. For the computation of the electromagnetic field distribution, the software package COMSOL Multiphysics is employed to solve Maxwell's 

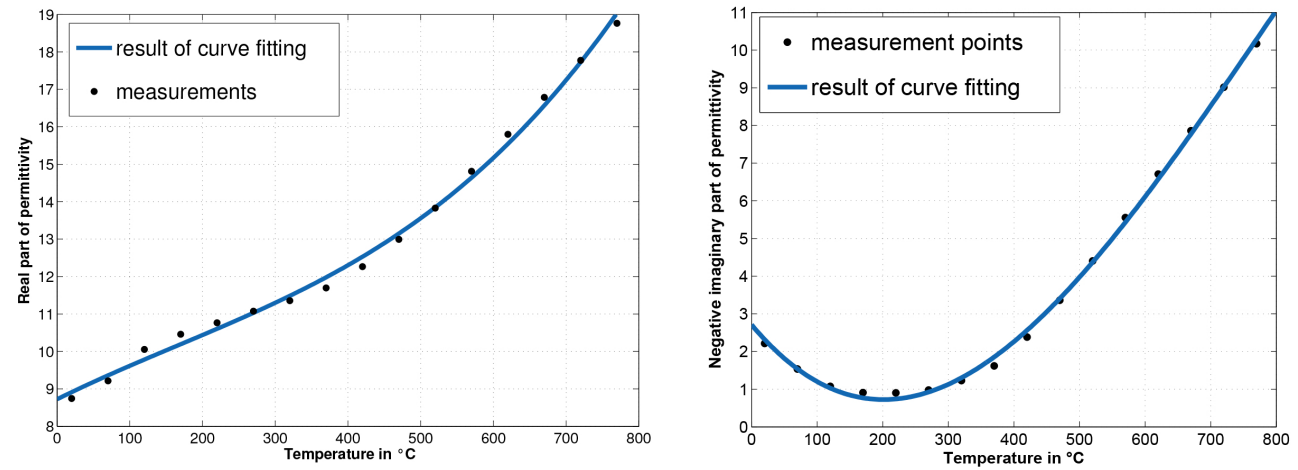

Figure 6. Results for the temperature-dependent, complex permittivity of the susceptor material silicon carbide obtained through resonator experiments.

equations (Maxwell, 1873), which are given by

$\nabla \times \boldsymbol{H}=\boldsymbol{J}+\frac{\partial \boldsymbol{D}}{\partial t} \quad$ (Ampère-Maxwell law)

$\nabla \times \boldsymbol{E}=-\frac{\partial \boldsymbol{B}}{\partial t} \quad$ (Faraday's law)

$\nabla \cdot \boldsymbol{B}=0 \quad$ (Gauss's law for magn. fields)

$\nabla \cdot \boldsymbol{D}=\rho_{e}, \quad$ (Gauss's law)

where $\boldsymbol{E}$ is the electric field, $\boldsymbol{H}$ the magnetic field, $\boldsymbol{D}$ the electric displacement current, $\boldsymbol{B}$ the magnetic flux density, $\rho_{\mathrm{e}}$ the electric charge density and $\boldsymbol{J}$ the electric current density. This set of equations is solved numerically by the finite element method (Jin, 2002). The resulting electromagnetic loss distribution acts as thermal heat source in a subsequent thermodynamic simulation. The solution of the heat conduction equation

$\rho_{\mathrm{m}} c \frac{\partial T}{\partial t}=\nabla(k \nabla T)+q$

provides the temperature distribution after a specified time $t$. Here, $\rho_{\mathrm{m}}$ denotes the mass density, $T$ the temperature, $k$ the thermal conductivity, $c$ the specific heat capacity and $q$ the absorbed power in a unit volume. The absorbed power can be written as

$q=2 \pi f \varepsilon_{0} \varepsilon_{\mathrm{r}}^{\prime \prime}\left|E_{\mathrm{eff}}\right|^{2}$

and depends on the frequency $f$, the permittivity of free space $\varepsilon_{0}$, the imaginary part of the relative permittivity $\varepsilon_{\mathrm{r}}^{\prime \prime}$ and the square of the effective value of the electric field $E_{\text {eff }}$.

In order to take into account the temperature dependency of the permittivity, electromagnetic and thermodynamic simulation have to be coupled. This means that after every time step of the transient thermal simulation, it has to be verified that the material parameters have not changed significantly due to the propagating heat. Each time the material parameters have altered to a certain degree, a new electromagnetic simulation has to be performed with updated material parameters according to the actual temperature distribution. The

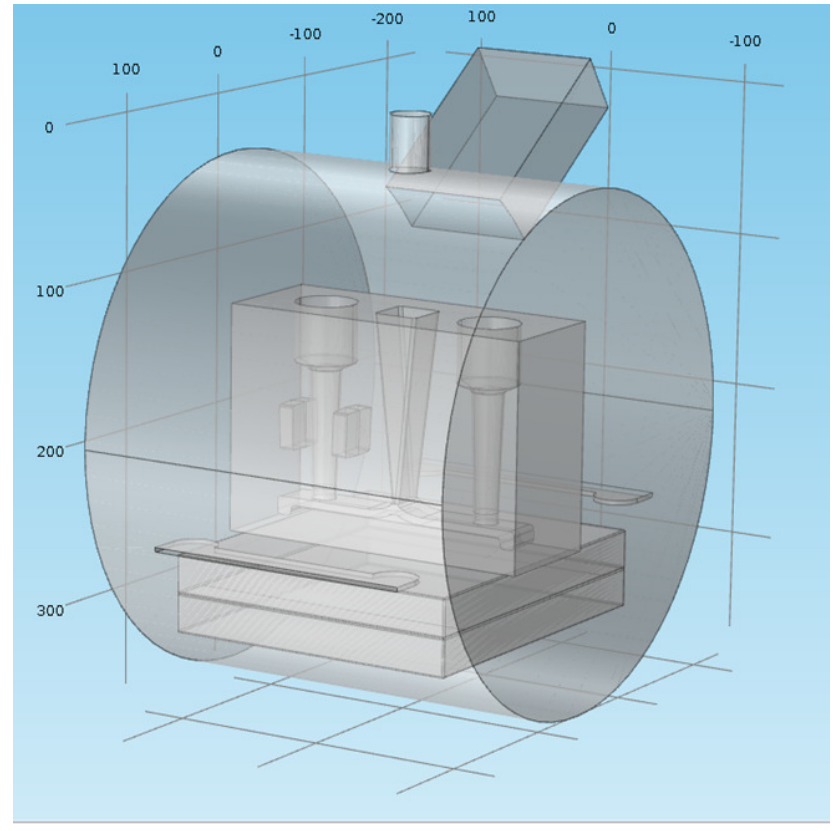

Figure 7. Simulation setup of a sand mold with susceptors inside the microwave chamber.

refreshed heat sources are then used for the next time steps of the transient thermal simulation, until the electromagnetic problem part has to be recomputed again.

This simulation setup is used to compute the expected temperature distribution inside a sand mold after a certain amount of time. In Fig. 7, a model of the experimental setup is shown which includes a sand mold with two susceptors inside a microwave chamber. The temperature distribution after $210 \mathrm{~s}$ of heating is illustrated in Fig. 8. It should be denoted that the sand mold absorbs almost no electromagnetic energy, whereas the susceptors heat up significantly. Moreover, it can be discerned that the heat is propagating well inside the sand mold, so that a potential influence on the solidification process of the cast metal is expected. The different temperature 


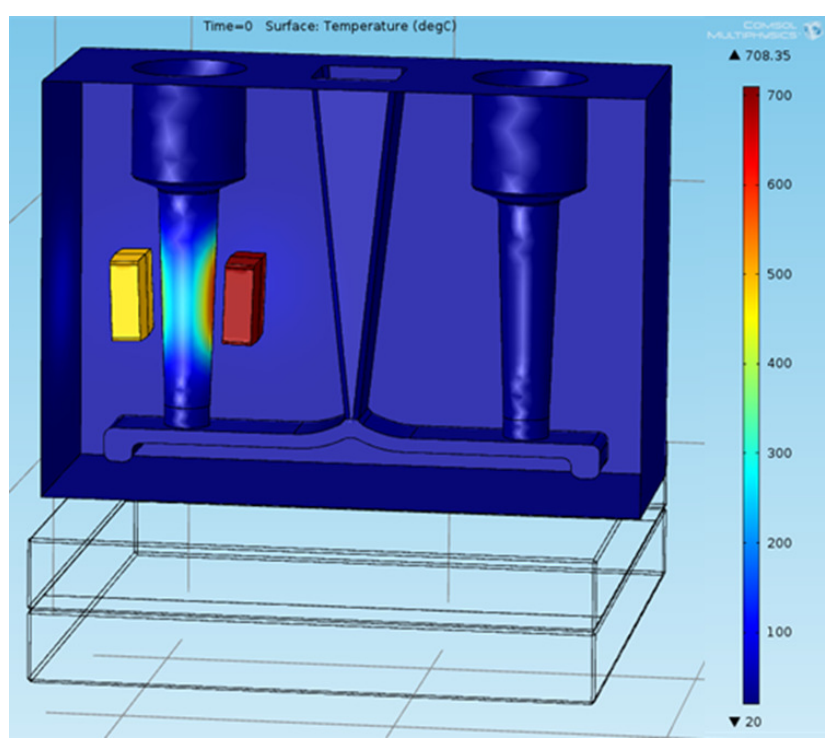

Figure 8. Temperature distribution inside sand mold after heating.

of the two susceptors is due to the inhomogeneous electric field. This issue can be addressed in future applications by embedding a mode stirring component (Wiedenmann et al., 2012).

\section{Experimental results}

\subsection{Comparison with simulation results}

To validate the simulation setup, numerical results are compared to results obtained from experimental measurements. For this purpose, the setup illustrated in Fig. 1 is adapted. Instead of a thermocouple, an infrared camera is used to measure the susceptor temperature during the heating phase. Here a $\mathrm{SiC}$ susceptor is embedded in a sand block which serves as susceptor carrier. Line of sight for the infrared camera is achieved by a cylindrical hole in the top part of the susceptor carrier. Microwave heating is applied for $90 \mathrm{~s}$ and the temperature is recorded using an infrared camera during heating and the subsequent cooling process. The measurement results are compared with the numerical results obtained from a simulation model with COMSOL Multiphysics in Fig. 9. Since the thermal conductivity of $\mathrm{SiC}$ and sand could not be measured, reference values obtained from literature were used instead. Silicon carbide shows rather high thermal conductivity, so that a possible error influences the results only negligibly. The considerably lower thermal conductivity of the sand effectively limits the heat transport away from the susceptor. Therefore, small variations in this parameter have a strong influence on the temperature characteristics. Whereas the thermal conductivity of the considered sand should be around $0.8 \mathrm{~W} \mathrm{~m}^{-1} \mathrm{~K}^{-1}$, a better agreement with the measurement curve can be observed if the thermal con- ductivity is decreased by $25 \%$. If the thermal conductivity was increased instead, the heat flow in the sand would be improved so that the surface temperature of the susceptor would be lowered.

For a closer matching of experimental results and numerical simulation, a more accurate knowledge of the thermophysical parameters such as thermal conductivity and specific heat capacity would be required.

\subsection{Casting experiments}

The described process of heating sand molds selectively by microwave energy gives us the possibility to generate a temperature distribution inside the mold prior to casting. Hereby we are able to influence the solidification characteristics of the aluminum alloy AlSi12 for example. For the casting experiments we manufactured molds from H32 and SLS sand with compressed $\mathrm{SiC}$ bound with INOTEC $®$ as susceptor material. Figure 10 shows an opened sand mold with the susceptors inside.

This mold is closed and placed into the microwave furnace for heating (see also Fig. 7). After that the aluminum alloy is cast into the mold's inlet at a temperature of approx. $750{ }^{\circ} \mathrm{C}$ and fills the specimen cavities from bottom to top. This way turbulent filling of the mold can be avoided. By casting into a series of these molds we could produce specimens which cooled naturally on the one side and were heated due to the localized susceptors on the other side of the mold. The cast specimens were cut in the center and analyzed with respect to their dendrite arm spacing (DAS). During solidification the primary aluminum phase forms tree-shaped arms, the so called dendrites. The DAS is defined as the distance between two dendrite arms and is a common metallographic parameter. Short solidification times result in small DAS and vice versa. Hereby we found, that specimens which cooled slower - since they were heated by the susceptors - had a larger DAS than naturally cooled ones. The results for different mold materials and heating times are shown in Fig. 11.

Although there is a relatively large deviation among the measurements of the DAS we could observe a clear trend that specimens which solidified and cooled near a susceptor had a considerable higher DAS. This effect could be shown with both mold materials and also with different heating times. Here longer heating times lead to a larger difference in DAS which is a consequence of the stronger heating in the susceptor area. These results correspond with the well-known link between solidification time and dendrite arm spacing and show that the solidification time of the aluminum alloy AlSi12 can be influenced by localized microwave heating. It should be noticed that DAS in this context is a measure for solidification time only. The connection of DAS and mechanical properties is not of interest in this case as the scope of the presented work is to overcome design limitations by locally manipulating solidification times. 


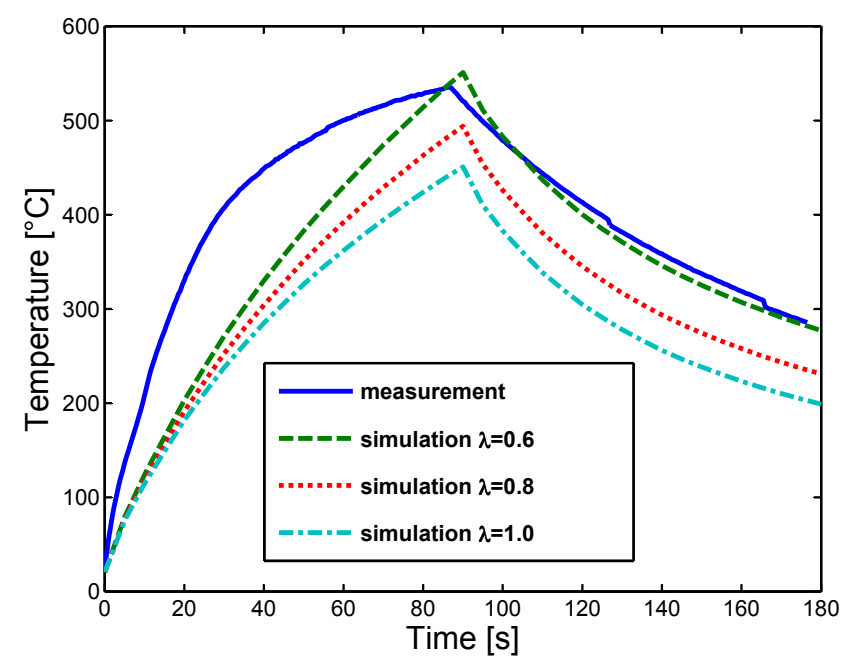

Figure 9. Comparison between experimental and simulation results for the setup shown in Fig. 1. The influence of the thermal conductivity of the sand is illustrated by considering the effect of an increase/decrease of the thermal conductivity by $25 \%$ on the temperature on the susceptor surface.
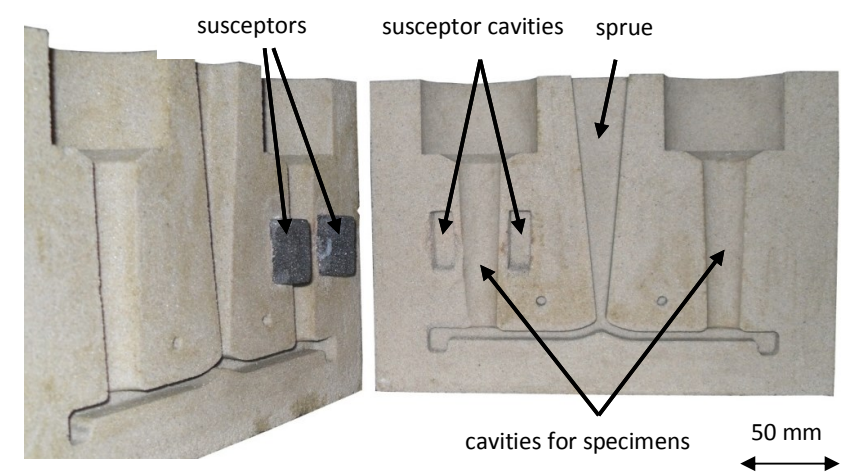

Figure 10. Opened sand mold with two inserted susceptors (black) for casting of two round specimens.

\section{Conclusions and outlook}

In this paper we presented a method for selectively heating a sand mold prior to a casting process. By using such a selectively heated sand mold it is possible to influence the thermal properties during the casting process and, hence, achieve desirable casing properties. The temperature field inside the sand mold is generated by a microwave process. The sand mold - which is mostly microwave transparent is locally equipped with susceptors, which strongly absorb the microwave energy and function as a heat source. Based on a selection of microwave absorbing materials we found compressed and chemically bound silicon carbide to be the most suitable material with respect to heating performance and mold stability. The susceptors were dielectrically characterized using a resonator technique and the temperature dependency was taken into account by parallel temperature

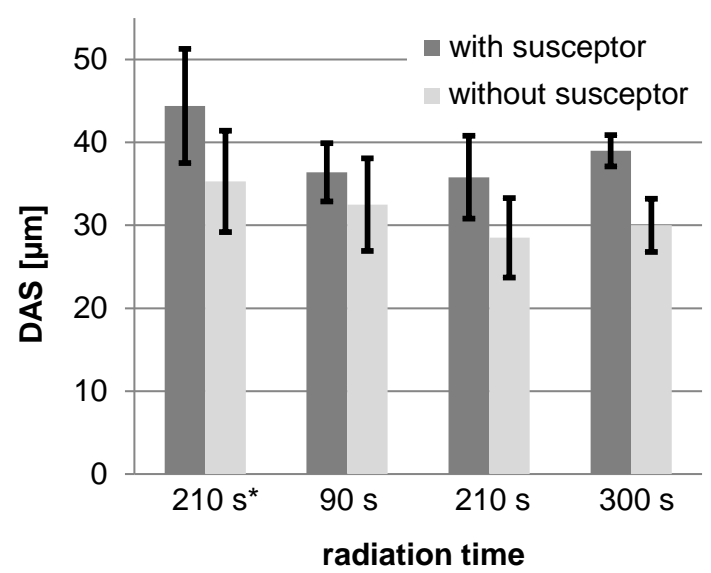

Figure 11. Dendrite Arm Spacing of specimens cast with (dark grey) and without (light grey) the heating influence of a susceptor for different radiation times. The column marked with * represents the only available experiment series with SLS molds. The three other displayed series were performed in H32 molds.

measurements with an infrared camera. A coupled electrothermal simulation model was created so that the temperature distribution inside a sand mold can be predicted and optimized. Comparisons between microwave heating experiments and numerical results showed fairly good agreement and first casting experiments confirmed the effect of local heating on the cooling process.

In future works, the electro-thermal simulation shall be directly coupled to a casting simulation, so that the whole process chain can be modeled and optimized.

Acknowledgements. The authors thank "Deutsche Forschungsgemeinschaft" (DFG) for partially funding this work under grant EI 352/9-2 and HO 2165/38-2.

Edited by: U. van Rienen

Reviewed by: M. H. Awida, T. Galek, and one anonymous referee

\section{References}

Bott, P.-M.: Untersuchungen zur Mikrowellenabsorption und zum Reduktionsverhalten oxidischer Systeme, Ph.D. thesis, Universität Tübingen, 1991.

Chandrasekaran, S., Basak, T., and Ramanathan, S.: Experimental and theoretical investigation on microwave melting of metals, J. Mater. Process. Tech., 211, 482-487, 2011.

Clark, D. E. and Folz, D. C.: Microwave Solutions for Ceramic Engineers, The American Ceramic Society, Westerville, 2005.

Gerdes, T., Willert-Porada, M., and Roediger, K.: Mikrowellensintern elektrisch leitfähiger Werkstoffe, Mikrowelleneinsatz in den Materialwissenschaften, der chemischen Verfahrenstechnik und in der Festkörperchemie, Shaker Verlag, Aachen, 1998.

Grellinger, D. J. and Janney, M. A.: Temperature measurement in a $2.45 \mathrm{GHz}$ microwave furnace, Ceramic Transactions Mi- 
crowaves: theory and applications in materials processing, Am. Ceram. Soc. Bull., 36, 529-538, Westerville, 1993.

Jin, J.: The Finite Element Method in Electromagnetics, John Wiley \& Sons, New York, NY, 2002.

Kılıç, E., Siart, U., Wiedenmann, O., Faz, U., Ramakrishnan, R., Saal, P., and Eibert, T. F.: Cavity resonator measurement of dielectric materials accounting for wall losses and a filling hole, IEEE T. Instrum. Meas., 62, 401-407, doi:10.1109/TIM.2012.2215072, 2013.

Lühken, A.: Ultraschall und Mikrowellenstrahlung im Chemieunterricht - Entwicklung und Erprobung einfacher Experimente zum nichtklassischen Energieeintrag, Ph.D. thesis, Johann W. Goethe-Universität Frankfurt a. Main, 2005.

Lukasz, R.: Mikrowellenunterstützte Wärme- und Stoffübertragung beim Trocknen und Entbindern technischer Keramik, Ph.D. thesis, TU Bergakademie Freiberg, 2005.

Maxwell, J. C.: A Treatise on Electricity and Magnetism, Oxford University Press, London, UK, 1873.
Meredith, R.: Engineers Handbook of Industrial Microwave Heating, The Institution of Electrical Engineers, London, England, 1998.

Rhee, S.: Mikrowellenprozesstechnik für keramische Werkstoffe der Mikrosystemtechnik, Ph.D. thesis, Forschungszentrum Karlsruhe GmbH, 2002.

Robak, L.: Mikrowellenunterstützte Wärme- und Stoffübertragung beim Trocknen und Entbindern technischer Keramik, Ph.D. thesis, Technische Universität Bergakademie Freiberg, Germany, 2005.

Wiedenmann, O., Ramakrishnan, R., Kılıç, E., Saal, P., Siart, U., and Eibert, T. F.: A multi-physics model for microwave heating in metal casting applications embedding a mode stirrer, Microwave Conference (GeMiC), 2012 The 7th German, Ilmenau, Germany, 12-14 March 2012, 1-4, 2012. 